DOI: 10.15593/2224-9877/2015.4.07

УДК 621.923

\author{
О.В. Захаров, А.И. Склярова \\ Саратовский государственный технический университет \\ им. Ю.А. Гагарина, г. Саратов, Россия \\ ГЕОМЕТРИЧЕСКИЕ И СИЛОВЫЕ АСПЕКТЫ \\ СУПЕРФИНИШИРОВАНИЯ ШАРОВ \\ АВИАЦИОННЫХ ПОДШИПНИКОВ
}

\begin{abstract}
Постоянно повышающиеся требования к качеству деталей авиационных и приборных подшипников обусловливают поиск новых технологий финишной обработки. В настоящее время в авиационных подшипниках применяют шары 20-й степени точности, у которых допуск на диаметр не превышает 1 мкм, сферичность - 0,5 мкм, волнистость - 0,05 мкм, шероховатость 0,02 мкм. Традиционная доводка шаров между двумя вращающимися дисками является малоэффективной в первую очередь с позиции производительности. В связи с этим научно и технически обосновано применение бесцентрового суперфиниширования шаров на станках с продольной подачей с использованием гладких валков. Экспериментально установлена принципиальная возможность применения подобной технологической системы для финишной обработки шаров подшипников. Основное преимущество данной схемы обработки заключается в высокой производительности и возможности полной автоматизации процесса. Вместе с тем требуется создать равномерное вращение заготовки шара в двух плоскостях в условиях силового замыкания контакта. Для этого решены ключевые геометрические и силовые задачи анализа условий стабильности силового замыкания контакта и профилирования валков фрормообразующей системы суперфинишного станка. Рассмотрена схема действия сил при суперфинишировании шаров и выявлено условие их движения с вращением в двух плоскостях. Разработана математическая модель фрормообразования и приведена методика расчета профиля валков численным методом. Приведенные результаты позволяют применять бесцентровое суперфиниширование шаров в качестве финишной операции вместо доводки, тем самым повысив производительность обработки в десятки раз.
\end{abstract}

Ключевые слова: бесцентровое суперфиниширование, авиационные подшипники, шары подшипников, профилирование, формообразование, геометрические расчеты, суперфинишный станок.

\author{
O.V. Zakharov, A.I. Skliarova
}

Yuri Gagarin State Technical University, Saratov, Russian Federation

\title{
GEOMETRIC AND STRENGTH ASPECTS OF SUPERFINISHING OF AIRCRAFT BEARINGS BALL
}

The steadily increasing demands on the quality of parts instrument and aircraft bearings are responsible for the search for new technologies finishing. Currently, aircraft bearings are used the balls 20 degree of precision in which the tolerance for the diameter is not greater than 1 micron, spherical$0.5 \mu \mathrm{m}$, waviness $-0.05 \mu \mathrm{m}$, roughness $-0.02 \mu \mathrm{m}$. Traditional finishing balls between two rotating disks is ineffective in the first place from the perspective of productivity. Therefore, scientific and technically justified 
application of centerless superfinishing balls on the machines with traverse with smooth rolls. It was established experimentally in principle the possibility of such a technological system for finishing ball bearings. The main advantage of this scheme processing is the high productivity and the possibility of complete automation of the process. However, the need to create a uniform rotation of the ball preform in two planes under power contact. For this solved the key problem of geometric and strength analysis of the conditions of stability of the power circuiting contact and profiling roll forming system superfinishing machine. The scheme of the forces in the superfinishing balls and revealed the condition of their movement with the rotation in two planes. A mathematical model of formation and describes the methodology for calculating the profile of the rolls by a numerical method. These results allow the use of centerless superfinishing balls as a finishing operation, instead of finishing, thereby increasing processing performance tenfold.

Keywords: centerless superfinishing, aircraft bearings, bearing balls, profiling, forming, geometric calculations, superfinishing machine.

Высокие требования к техническим характеристикам приборов и изделий авиационно-космической техники обусловливают необходимость совершенствования конструкций, применения новых материалов, повышения точности размеров и формы деталей. Например, в авиационных подшипниках применяют шары 20-й степени точности, у которых допуск на диаметр не превышает 1 мкм, сферичность 0,5 мкм, волнистость - 0,05 мкм, шероховатость - 0,02 мкм. С целью выполнения указанных требований разработаны шары из керамических и композиционных материалов [1], а также полые тонкостенные шары [2]. Еще более жесткие требования предъявляются к бериллиевым роторам гироскопов с электростатическим подвесом [3].

Изготовление шаров в подшипниковой промышленности традиционно реализуется в виде доводки между двумя чугунными дисками с кольцевыми канавками [4-7]. Такая технология обеспечивает высокую точность формы и малую шероховатость поверхности, но имеет низкую производительность и способствует формированию дефектного слоя с неблагоприятным распределением остаточных напряжений [8-11].

Попытки решить данную проблему привели к созданию схемы бесцентрового шлифования шаров на станках с продольной подачей [12]. В отличие от известной схемы обработки цилиндрических заготовок ведущий круг был выполнен с винтовой канавкой несимметричного профиля, а шлифовальный круг снабжен кольцевыми канавками. Такое решение обеспечило вращение шара от сил трения с ведущим кругом и дополнительное верчение за счет периодического прерывания контакта со шлифовальным кругом. Основное преимущество разработанного метода обработки заключается в высокой производительности процесса. Вместе с тем точечный контакт заготовки со шлифовальным кругом и малое время контакта, лимитируемое длиной кругов, шагом винтовой канавки и частотой вращения ведущего круга, 
не позволяют достичь малой волнистости и шероховатости поверхности обрабатываемых шаров, поэтому целесообразным представляется введение последующей финишной обработки абразивными брусками.

Основой для настоящих исследований стал опыт зарубежных производителей, в первую очередь в Японии, по бесцентровому суперфинишированию шаров на станках с продольной подачей. Для практической реализации требуется комплексное решение задач по профилированию валков, наладке станка, назначению рациональных режимов резания. Одной из ключевых задач становится установление силовых условий формообразования сфер, которые затем будут положены в основу методики наладки станков. До настоящего времени данная задача не нашла теоретического решения в известной литературе [13].

Геометрически правильное формообразование сферической поверхности шара может быть обеспечено только при равномерном съеме припуска шлифовальным бруском со всей площади обрабатываемой поверхности заготовки. В процессе обработки произвольно выбранная точка на поверхности шара движется по сложной пространственной траектории, которая может быть определена при известной скорости этой точки в любой момент времени. Заготовка приводится в движение за счет сил трения в результате равномерного вращения вокруг неподвижных перекрещивающихся осей двух валков станка. Скорость вращения, межосевое расстояние и угол между осями вращения представляют собой параметры наладки оборудования, поэтому траектория движения центра масс шара есть функция, определяемая профилем валков и параметрами геометрической наладки валкового устройства, получаемыми из решения геометрической задачи формообразования [14].

Для определения движения произвольной точки шара в данный момент времени, согласно теореме Шаля [15], достаточно знать направление скорости одной из его точек и абсолютную величину и направление другой его точки. В качестве первой точки можно принять центр масс шара, поскольку его траектория и касательная, совпадающая с вектором скорости, известны. В качестве второй точки может быть взята точка контакта шара с валком, поскольку линейная скорость валка, обусловленная известными значениями его радиуса и угловой скорости вращения, может быть определена. Для расчета линейной скорости точки контакта шара также необходимо знать коэффициент передачи скорости от валка. Применим для решения практической 
задачи допущение, что один из валков передает движение шару без проскальзывания, т.е. векторы линейной скорости валка и шара в точке контакта совпадают. Назовем данный валок ведущим. Тогда по другому валку, вследствие различия радиусов валков при равенстве их угловых скоростей вращения в плоскости контакта, шар будет проскальзывать. Назовем данный валок опорным. Под плоскостью контакта понимаем плоскость, проходящую через точки контакта шара с валками и центром $X 0 Y$ (рис. 1, a). Касательная к траектории, совпадающая с вектором скорости в центре 0 масс, будет перпендикулярна этой плоскости.

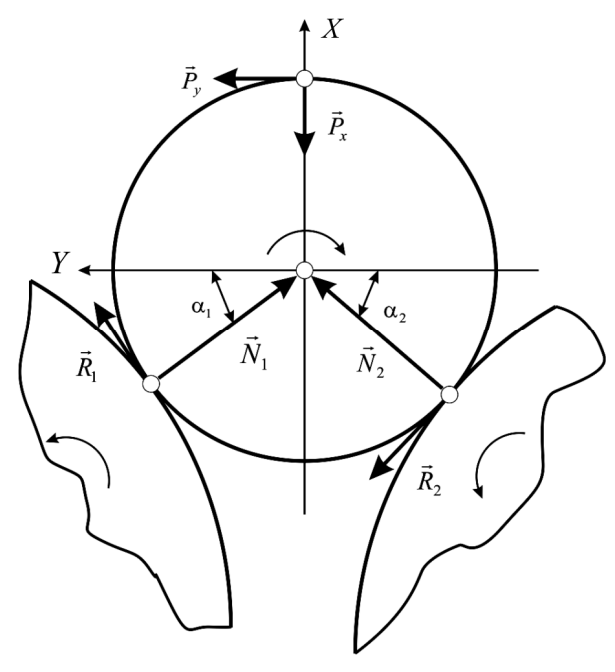

$a$

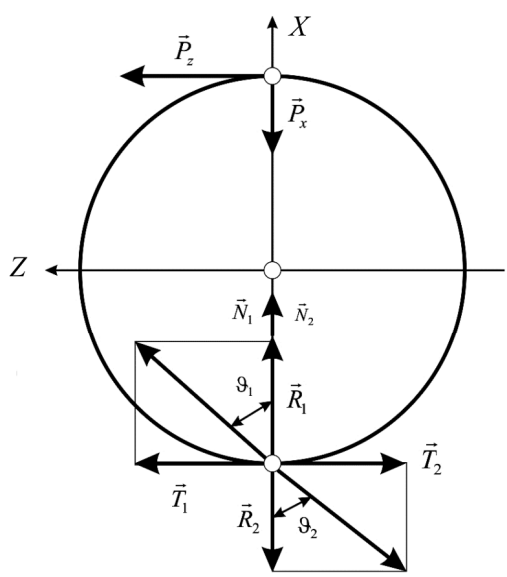

$\sigma$

Рис. 1. Силовая схема бесцентрового суперфиниширования шара:

$a$ - поперечная секущая плокость; $\sigma$ - продольная секущая плоскость

Для определения скорости точки контакта шара с валками необходимо знать, какой из них будет ведущим. Для этого требуется решение задачи силового замыкания контакта шара, валков и шлифовального бруска. Введем ряд предположений и допущений:

1) центр 0 масс шара движется равномерно прямолинейно вдоль оси $0 Z$ параллельно траектории осцилляции шлифовальных брусков;

2) сила резания раскладывается на три ортогональные составляющие $P_{x}, P_{y}, P_{z}$, параллельные выбранным соответствующим осям координат;

3) шар приводится в движение только ведущим валком, другой валок выступает в качестве опорного;

4) ведущий валок определяется условием отсутствия проскальзывания в точке контакта с шаром, т.е. для него выполняется условие $F_{\text {тр }}<f N$; 
5) влияние моментов трения верчения и качения шара в точках контакта с валками исключено из рассмотрения, поскольку для определения этих величин необходимо решить задачу по определению деформации в точках контакта. Это существенно усложняет данную задачу, давая лишь незначительный для практики выигрыш в точности результата;

6) угловая скорость вращения ведущего валка постоянна, поэтому проекция линейной скорости валка в точке контакта на плоскость $X 0 Y$ тоже постоянна. Следовательно, вращение шара вокруг оси $0 Z$, обусловленное этой скоростью, будет равномерным, а крутящий момент сил при постоянной угловой скорости вокруг оси $0 Z$ будет равен нулю.

Опираясь на первое и шестое положения, действие сил на шар со стороны валков и шлифовальных брусков можно описать четырьмя уравнениями статики. Для крутящих моментов относительно осей $0 X$ и $0 Y$ уравнения не записываются в силу пятого положения.

Составим систему уравнений статики для случая, когда шар выйдет из состояния покоя или чистого качения и начнет проскальзывать по обоим валкам:

$$
\left\{\begin{array}{l}
\sum X=0 \rightarrow N_{1} \sin \alpha_{1}+N_{2} \sin \alpha_{2}+R_{1} \cos \alpha_{1}-R_{2} \cos \alpha_{2}-P_{x}=0, \\
\sum Y=0 \rightarrow N_{2} \cos \alpha_{2}-N_{1} \cos \alpha_{1}+R_{1} \sin \alpha_{1}+R_{2} \sin \alpha_{2}+P_{y}=0 \\
\sum Z=0 \rightarrow T_{1}-T_{2}+P_{z}=0 \\
\sum M_{0 Z}=0 \rightarrow R_{1} r+R_{2} r-P_{y} r=0
\end{array}\right.
$$

где $N_{1}, N_{2}$ - силы нормальной реакции в точках контакта 1 и $2 ; F_{1}, F_{2}-$ силы трения в точках контакта шара с валками; $R_{1}, R_{2}-$ проекция сил трения $F_{1}$ и $F_{2}$ на плоскость $X 0 Y ; T_{1}, T_{2}$ - проекции силы трения на ось $0 Z ; P_{x}, P_{y}, P_{z}-$ проекции силы резания на оси $X, Y, Z ; \alpha_{1}, \alpha_{2}, \vartheta_{1}, \vartheta_{2}-$ углы контакта и разворота силы трения $F_{1}$ и $F_{2}$ в точках 1 и 2 соответственно; $r$ - радиус шара.

Преобразуя систему (1), получим

$$
\left\{\begin{array}{l}
N_{1} \sin \alpha_{1}+N_{2} \sin \alpha_{2}+f_{1} N_{1} \cos \vartheta_{1} \cos \alpha_{1}-f_{2} N_{2} \cos \vartheta_{2} \cos \alpha_{2}-P_{x}=0 \\
N_{2} \cos \alpha_{2}-N_{1} \cos \alpha_{1}+f_{1} N_{1} \cos \vartheta_{1} \sin \alpha_{1}+f_{2} N_{2} \cos \vartheta_{2} \sin \alpha_{2}+P_{y}=0 \\
f_{1} N_{1} \sin \vartheta_{1}-f_{2} N_{2} \sin \vartheta_{2}+P_{z}=0 \\
f_{1} N_{1} \cos \vartheta_{1}+f_{2} N_{2} \cos \vartheta_{2}-P_{y}=0
\end{array}\right.
$$

где $f_{1}$ и $f_{2}-$ коэффициенты трения. 
Поскольку искомые углы $\vartheta_{1}, \vartheta_{2}$ входят в уравнения системы (2) как аргументы тригонометрических функций, то решение находится численными методами, например путем минимизации целевой функции $\Phi$, представляющей собой сумму $v_{i}$ квадратов левых частей уравнений системы (2):

$$
\Phi\left(\vartheta_{1}, \vartheta_{2}, N_{1}, N_{2}\right)=v_{1}^{2}+v_{2}^{2}+v_{3}^{2}+v_{4}^{2} \rightarrow \min .
$$

Определим условия, при которых ведущим будет первый валок (слева на рис. 1). Для этого зададим условие $F_{1}<f N_{1}$, введя произвольно взятый коэффициент $0<k<1$ для $f_{1}=k f_{2}$. Тогда для первого валка выполняется условие, соответствующее трению покоя или чистого качения (без проскальзывания), $F_{1}=f_{1} N_{1}=k f N_{1}<f N$, а для второго валка выполняется условие, соответствующее трению скольжения, $f_{2}=f$, $F_{2}=f_{2} N_{2}=f N$. По соотношению сил $N_{1}$ и $N_{2}$ можно заключить, что на первый валок действует бо́льшая по величине прижимная сила и именно он будет ведущим.

Другой из необходимых теоретических задач при бесцентровом суперфинишировании шаров будет расчет профиля валков. Предлагаемый подход к теоретическому описанию процесса формообразования основан на том, что форма заготовки однозначно формируется траекторией ее движения относительно шлифовальных брусков станка. Для профилирования валков использован численный метод решения, так как традиционный метод исследования пространственных зацеплений, построенный на векторно-матричных преобразованиях координат и кинематическом условии огибания поверхностей, не позволяет определить все неизвестные параметры.

Обработка ведется на серийных станках моделей ВШ-774, SZZ-3 с использованием гладких валков сложной формы наружной поверхности. При установке угла разворота валков в интервале $0,5-1^{\circ}$ время обработки составляет от 50 до 100 с. За счет правильного выбора наладочных параметров станка может быть обеспечено равномерное вращение шара и его верчение в разных плоскостях, при котором сетка рисок достаточно равномерно покрывает поверхность шара [9].

Введем в рассмотрение следующие координатные системы (рис. 2): $S_{0}\left(X_{0} 0_{0} Y_{0} Z_{0}\right)$ - условно неподвижная система станка; $S_{1}\left(X_{1} 0_{1} Y_{1} Z_{1}\right)$ система заготовки шара; $S_{2}\left(X_{2} O_{2} Y_{2} Z_{2}\right)$ - система левого валка. Система координат $S_{2}$ по отношению к системе $S_{0}$ повернута вокруг оси $Y$ по 
часовой стрелке на угол $\lambda$ (половина угла перекрещивания осей валков) и смещена по оси $X$ на величину $h$ (наладочное смещение) и по оси $Y$ на величину $v$ (половина кратчайшего межосевого расстояния валков). Система координат $S_{1}$ по отношению к системе $S_{0}$ смещена по оси $Z$ на величину $a$.

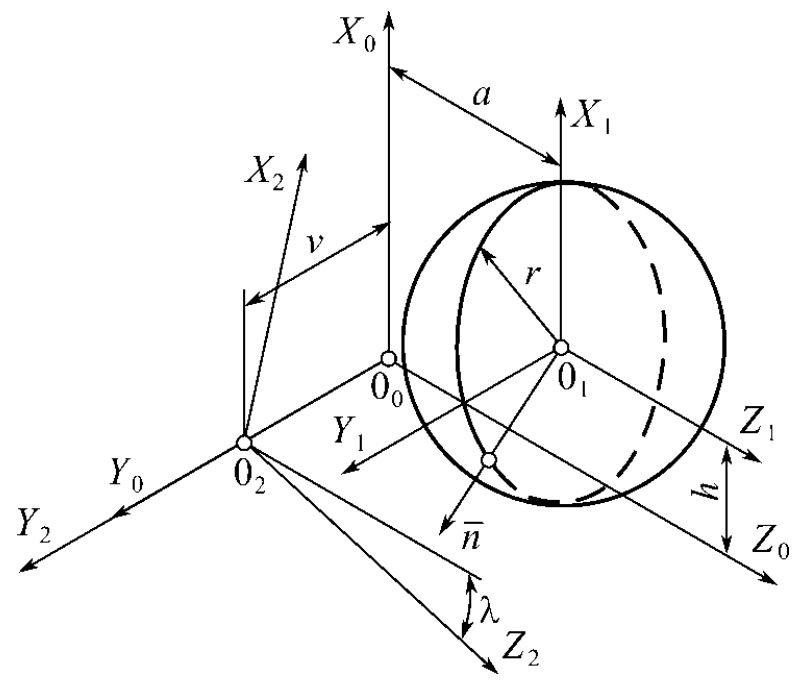

Рис. 2. Координатная схема профилирования валков

Сферическая поверхность заготовки в системе $S_{0}$ описывается уравнениями

$$
\left.\begin{array}{l}
X_{0}=-r \sin \beta, \\
Y_{0}=r \cos \alpha \cos \beta, \\
Z_{0}=r \sin \alpha \cos \beta,
\end{array}\right\}
$$

где $r$ - радиус сферы; $\alpha, \beta$ - независимые угловые координаты сферы.

Выполнив последовательно преобразования уравнений (4) из системы $S_{1}$ в $S_{0}$, а затем в $S_{2}$, получим выражения для семейства поверхностей сферы в относительном движении:

$$
\left.\begin{array}{l}
X_{2}=A \cos \lambda-B \sin \lambda, \\
Y_{2}=r \cos \alpha \cos \beta-v, \\
Z_{2}=A \sin \lambda+B \cos \lambda,
\end{array}\right\}
$$

где $A=h-r \sin \beta ; B=r \sin \alpha \cos \beta+a, a-$ смещение сферы вдоль валков относительно проекции точки перекрещивания их осей. 
Условие пересечения нормалей к поверхности сферы с осью валка, составленное в системе $S_{0}$, имеет вид

$$
\left|\begin{array}{ccc}
h-r \sin \beta & r \cos \alpha \cos \beta-v & r \sin \alpha \cos \beta+a \\
-\sin \beta & \cos \alpha \cos \beta & \sin \alpha \cos \beta \\
\sin \lambda & 0 & \cos \lambda
\end{array}\right|=0 .
$$

Таким образом, окончательно поверхность валка описана уравнениями (4)-(6). Профиль валка как тела вращения целесообразно задавать координатами $\left(Z_{2}, R_{2}\right)$, где $R_{2}=\sqrt{X_{2}^{2}+Y_{2}^{2}}$. Однако в этом случае число неизвестных параметров превышает число составленных уравнений, поэтому требуется численный метод решения. Алгоритм численного профилирования валков состоит в следующем. Задается сечение валка $Z_{2}=$ const, и из последнего уравнения системы (2) после подстановки в него решения уравнения (3) находится точка сферы, нормаль в которой пересекает ось валка при некотором фиксированном значении $a$. Определяются координаты $\left(Z_{2}, R_{2}\right)$. Изменяя значения параметра $a$ в некотором интервале вокруг сечения $Z_{2}=$ const, получаем набор точек контакта сферы и валка в их относительном движении. Профилирующей будет та из них, которая имеет наименьший радиус $R_{2}$.

Расчеты показали, что левый и правый валки имеют одинаковый профиль, но так как развернуты в противоположных направлениях, то симметричны относительно плоскости $Z_{2}=0$. Поверхность валков имеет форму, близкую к поверхности однополостного гиперболоида вращения с несколько меньшей кривизной. В зависимости от принятых наладочных параметров меняется кривизна профиля валка.

Таким образом, теоретически обоснована возможность формообразования сферических поверхностей шаров на бесцентровых суперфинишных станках с продольной подачей. Анализ схемы действия сил при бесцентровом супефинишировании сфер позволил выявить, что ведущий валок определяется направлением вращения валков. Полученное условие силового замыкания контакта может быть использовано для проверки принятых значений наладочных параметров суперфинишного станка. Рассмотренный численный метод профилирования позволяет определить профиль валков с достаточной для практики точностью.

Работа выполнена при финансовой поддержке гранта Президента РФ (МД-1377.2014.8). 


\section{Список литературы}

1. Керамические тела качения перспективных шарикоподшипников: материал, технология изготовления и механическая обработка, расчеты и испытания / Т.Д. Каримбаев, М.А. Мезенцев, А.И. Алферов, С.К. Гордеев // Композиты и наноструктуры. - 2010. - № 2. - С. 12-27.

2. Васин А.Н., Бочкарев П.Ю. Получение заготовок полых тел качения // Технология машиностроения. - 2004. - № 4. - С. 3-6.

3. Requirements for MEMs GURO shock tests / M.I. Evstifeev, D.P. Eliseev, D.V. Rozentsvein, I.B. Chelpanov // Gyroscopy and Navigation. - 2013. - Vol. 3, iss. 1. - P. 51-55.

4. Олендер Л.А. Технология и оборудование шарикового производства. - Минск: Выш. шк., 1974. - 334 с.

5. Ящерицын П.И. О механизме формообразования шариков при их доводке // Подшипниковая промышленность. - 1977. - Вып. 4. - С. 18-27.

6. Бухаркин Л.Н., Иногамов Т.И. Доводка крупных шариков повышенной точности // Известия вузов. Машиностроение.- 1972.№ 1. - С. 172-177.

7. Клебанов М.К., Малахов А.Ф., Шабанов Л.А. Новый метод доводки шариков подшипников // Станки и инструмент. - 1975. - № 10. С. 28-29.

8. Куранов В.Г., Виноградов А.Н. Явление «трибоцементации» в процессе финишной обработки шаров подшипников // Трение и смазка в машинах и механизмах. - 2007. - № 8. - С. 32-38.

9. Теоретическая модель прогнозирования остаточных напряжений при шлифовании / А.М. Долганов, В.А. Жуланов, Т.Н. Иванова, Ф.В. Юрков // Науч.-техн. вестник Поволжья. - 2012. - № 3. - С. 110-113.

10. Технологическое обеспечение трибологических свойств сальниковых шеек деталей машин / И.Н. Бобровский, П.А. Мельников, Н.М. Бобровский, А.А. Лукьянов, А.В. Ежелев // Известия Самар. науч. центра РАН. - 2012. - Т. 14, № 1-2. - С. 340-343.

11. Шрубченко И.В., Рыбалко В.Ю. Оптимизация режимов резания для обработки поверхностей качения опор технологических барабанов // Технология машиностроения. - 2013. - № 8. - С. 12-16.

12. Васин А.Н., Изнаиров Б.М., Бочкарев А.П. Взаимодействие заготовки со шлифовальным кругом при бесцентровом шлифовании шариков // СТИН. - 2013. - № 7. - С. 18-21.

13. Мазальский В.Н. Суперфинишные станки. - Л.: Машиностроение, 1988. - 127 с. 
14. Zakharov O.V. Principles for the adjustment of centerless superfinishing machines // Russian Engineering Research. - 2011.- Vol. 31, iss. 5. - P. 465-468.

15. Бухгольц Н.Н. Основной курс теоретической механики. Ч. 1. Кинематика, статика, динамика материальной точки. - М.: Наука, 1967. -468 c.

\section{References}

1. Karimbaev T.D., Mezentsev M.A., Alferov A.I., Gordeev S.K. Keramicheskie tela kacheniia perspektivnykh sharikopodshipnikov: material, tekhnologiia izgotovleniia i mekhanicheskaia obrabotka, raschety i ispytaniia [Ceramic rolling element bearings perspective: the material, manufacturing technology and machining, calculations and tests]. Kompozity i nanostruktury, 2010, no. 2, pp. 12-27.

2. Vasin A.N., Bochkarev P.Iu. Poluchenie zagotovok polykh tel kacheniia [Getting blanks hollow rolling bodies]. Tekhnologiia mashinostroeniia, 2004, no. 4, pp. 3-6.

3. Evstifeev. M.I., Eliseev D.P., Rozentsvein D.V., Chelpanov I.B. Requirements for MEMs GURO shock tests. Gyroscopy and Navigation, 2013, vol. 3, iss. 1, pp. 51-55.

4. Olender L.A. Tekhnologiia i oborudovanie sharikovogo proizvodstva [Technology and equipment for the production of ball]. Minsk: Vysheishaia shkola, 1974. $334 \mathrm{p}$.

5. Iashcheritsyn P.I. O mekhanizme formoobrazovaniia sharikov pri ikh dovodke [On the mechanism of formation of balls in their finishing]. Podshipnikovaia promyshlennost', 1977, iss. 4, pp. 18-27.

6. Bukharkin L.N., Inogamov T.I. Dovodka krupnykh sharikov povyshennoi tochnosti [Lapping large balls of high accuracy]. Izvestiia vuzov. Mashinostroenie, 1972, no. 1, pp. 172-177.

7. Klebanov M.K., Malakhov A.F., Shabanov L.A. Novyi metod dovodki sharikov podshipnikov [A new method for finishing the bearing balls]. Stanki i instrument, 1975, no. 10. pp. 28-29.

8. Kuranov V.G., Vinogradov A.N. Iavlenie "tribotsementatsii" v protsesse finishnoi obrabotki sharov podshipnikov [The phenomenon of "tribochemistry" in the process of finishing the bearing balls]. Trenie i smazka $v$ mashinakh i mekhanizmakh, 2007, no. 8, pp. 32-38. 
9. Dolganov A.M., Zhulanov V.A., Ivanova T.N., Iurkov F.V. Teoreticheskaia model' prognozirovaniia ostatochnykh napriazhenii pri shlifovanii [The theoretical model for predicting residual stresses during grinding]. Nauchno-tekhnicheskii vestnik Povolzh'ia, 2012, no. 3, pp. 110-113.

10. Bobrovskii I.N., Mel'nikov P.A., Bobrovskii N.M., Luk'ianov A.A., Ezhelev A.V. Tekhnologicheskoe obespechenie tribologicheskikh svoistv sal'nikovykh sheek detalei mashin [Technological support tribological properties necks packing machine parts]. Izvestiia Samarskogo nauchnogo tsentra Rossiiskoi akademii nauk, 2012, vol. 14, no. 1-2, pp. 340-343.

11. Shrubchenko I.V., Rybalko V.Iu. Optimizatsiia rezhimov rezaniia dlia obrabotki poverkhnostei kacheniia opor tekhnologicheskikh barabanov [Optimization of cutting conditions for surface treatment technology supports rolling drums]. Tekhnologiia mashinostroeniia, 2013, no. 8, pp. 12-16.

12. Vasin A.N., Iznairov B.M., Bochkarev A.P. Vzaimodeistvie zagotovki so shlifoval'nym krugom pri bestsentrovom shlifovanii sharikov [Interaction of the workpiece with the grinding wheel centerless grinding balls]. Stanki. Instrument, 2013, no. 7, pp. 18-21.

13. Mazal'skii V.N. Superfinishnye stanki [Superfinishing machines]. Leningrad: Mashinostroenie, 1988. 127 p.

14. Zakharov O.V. Principles for the adjustment of centerless superfinishing machines. Russian Engineering Research, 2011, vol. 31, iss. 5, pp. 465-468.

15. Bukhgol'ts N.N. Osnovnoi kurs teoreticheskoi mekhaniki. Chast' 1. Kinematika, statika, dinamika material'noi tochki [The main course of theoretical mechanics. Part 1. Kinematics, statics, dynamics of a material point]. Moscow: Nauka, 1967. 468 p.

Получено 28.10.2015

\section{Об авторах}

Захаров Олег Владимирович (Саратов, Россия) - доктор технических наук, доцент, профессор кафедры «Проектирование технических и технологических комплексов» Саратовского государственного технического университета им. Ю.А. Гагарина; e-mail: zov20@mail.ru.

Склярова Анастасия Игоревна (Саратов, Россия) - магистрант кафедры «Теплогазоснабжение, вентиляция, водообеспечение и при- 
кладная гидрогазодинамика» Саратовского государственного технического университета им. Ю.А. Гагарина.

\begin{abstract}
About the authors
Oleg V. Zakharov (Saratov, Russian Federation) - Doctor of Technical Sciences, Professor of Department "Designing and Technological Complexes", Yuri Gagarin State Technical University; e-mail: zov20@mail.ru.

Anastasia I. Skliarova (Saratov, Russian Federation) - Master Student, Department "Heat Supply, Ventilation, Water Supply and Applied Fluid Dynamics", Yuri Gagarin State Technical University.
\end{abstract}

